\section{FRI0355 IL-17A UP-REGULATION IN PERIPHERAL BLOOD MONONUCLEAR CELLS CO-CULTURED WITH AUTOLOGOUS SKIN FIBROBLASTS IS ASSOCIATED WITH DOWN-REGULATION OF PRO-FIBROTIC MEDIATORS AND INCREASED FIBROBLAST APOPTOSIS}

S. Vettori ${ }^{1}$, G. Barra ${ }^{2}$, G. Pasquale ${ }^{3}$, L. Pellecchia ${ }^{1}$, L. Vicedomini ${ }^{1}$, R. De Palma ${ }^{1}$, G. Valentini ${ }^{1} .1$ Internal and Experimental Medicine "F. Magrassi", University of Campania "Luigi Vanvitelli", Naples; ${ }^{2}$ Internal and Experimental Medicine "F. Magrassi", University of Campania "Luigi Vanvitelli", Napoli; ${ }^{3}$ Internal and Experimental Medicine "F. Magrassi", University of Campania "Luigi Vanvitelli", Naples, Italy

Background: IL-17A has been implicated in the pathogenesis of systemic sclerosis (SSc) (1). We previously showed that skewed peripheral blood mononuclear cells (PBMCs) from SSc patients can induce Fas-mediated apoptosis in co-cultured autologous skin fibrobalsts (2).

Objectives: We therefore aimed to investigate IL-17A expression and effects in these co-cultures.

Methods: PBMCs and skin fibroblasts from 5 dcSSc patients with disease duration $<3$ years were co-cultured up to 10 days in presence of hrlL-2 $[20 \mathrm{U} / \mathrm{ml}]$ in a 1:10 ratio, as previously described. IL17A, IL17RA, CXCL1, CCL2, CCL3, TGFBR2, SMAD3, CTGF, COL1A1, COL3A1 mRNA expression was assessed by Sybr Green real-time PCR. Chemokine production was further investigated at the protein level by multiple suspension immunoassay. In subset experiments, co-cultures were treated with either IL-17A or IL-17A plus anti-IL17 receptor A neutralizing monoclonal antobodies (anti-IL-17RA mAb), then cells were stained with Annexin V, anti-IL17RA, and anti-FAS antibodies and were investigated by flow-citometry.

Results: IL17A mRNA in co-cultured PBMCs was increased by 11.5 fold $(p<0.01)$, and IL17RA by 4.3 fold $(p<0.05)$ in co-cultured fibroblasts. CXCL-11, CCL2, and CCL3 were also up-regulated at both mRNA (11.9 fold, 773.3 fold, and 29 fold, respectively; $p<0.05)$ and protein level $(8.9$ fold, 11.2 fold, and 252.4 fold, respectively; $p<0.05)$. Profibrotic mediators, such as COL1A1, COL3A1, and CTGF mRNA expression in co-cultured fibroblasts was reduced to 0.33 fold, 0.24 fold, and 0.31 fold, respectively $(p<0.05)$. This effects were associated with mRNA down-regulation of two key effectors of TGF- $\beta$ signaling, TGFBR2 and SMAD3 to 0.59 and 0.79 fold, respectively. At flow cytometry analysis, we observed a reduction in co-cultured fibroblasts apoptosis by adding IL-17RA neutralizing $\mathrm{mAb}$ to IL-17A treated cells ( $39 \%$ to $16.8 \% ; p<0.05)$, as compared to controls treated with IL-17A and isotype controls. Moreover, IL17RA mAb addition also reduced Fas expression in co-cultured fibroblasts as compared to IL-17A treated cells $(47.7 \%$ to $10.6 \% ; p<0.05)$.

Conclusions: Our results support the role of IL-17A in the pathogenesis of SSc. Furthermore, here we first show that IL-17A up-regulation in co-cultured PBMCs might play antifibrotic effects in autologous skin fibroblasts and might be implicated in fibroblast apoptosis, interphering with the FAS/FASL pathway.

\section{References:}

[1] Brembilla NC, Chizzolini C. T cell abnormalities in systemic sclerosis with a focus on Th17 cells. Eur Cytokine Netw 2012; 23: 128-39.

[2] De Palma R et al. Peripheral T cells from patients with early systemic sclerosis kill autologous fibroblasts in co-culture: is T-cell response aimed to play a protective role? Rheumatology (Oxford) 2010; 49: 1257-66.

Disclosure of Interest: None declared

DOI: 10.1136/annrheumdis-2017-eular.6051

\section{FRI0356 ANTISENSE LONG NONCODING RNAS ARE DEREGULATED IN SKIN TISSUE OF SSC PATIENTS}

T. Messemaker ${ }^{1}$, L. Chadli ${ }^{2}$, G. Cai ${ }^{3}$, V. Goelela ${ }^{2}$, M. Boonstra ${ }^{1}$, A. Dorjee ${ }^{4}$ S. Andersen ${ }^{2}$, H. Mikkers ${ }^{4}$, T. Huizinga ${ }^{4}$, Z. Li ${ }^{3}$, O. Distler ${ }^{5}$, M. Whitfield ${ }^{3}$, R. Toes ${ }^{4}$, J. Aarbiou ${ }^{2}$, J. De Groot ${ }^{2}$, J. De Vries-Bouwstra ${ }^{4}$, F. Kurreeman ${ }^{4}$ ${ }^{1}$ Rheumatology, LUMC: ${ }^{2}$ Charles River Lab, Leiden, Netherlands: ${ }^{3}$ Geisel School of Medicine at Dartmouth, Hanover, United States; ${ }^{4}$ LUMC, Leiden, Netherlands; ${ }^{5}$ University Hospital Zurich, Zurich, Switzerland

Background: Systemic sclerosis (SSc) is an autoimmune disease characterized by fibrosis of skin and multiple organs of which pathogenesis is poorly understood. Here we studied differentially expressed coding and non-coding genes in relation to SSc pathogenesis with a specific focus on antisense non-coding RNAs.

Objectives: Here we studied differentially expressed coding and non-coding genes in relation to SSc pathogenesis with a specific focus on antisense non-coding RNAs.

Methods: Skin biopsy-derived RNAs from fourteen early SSc patients and six healthy individuals were sequenced with ion-torrent and analysed using DEseq2. Protein-coding and non-coding genes annotated in GENCODEV7 were analysed. Significant long non-coding RNAs were independently replicated in a Northern American dataset.

Results: 4901 genes with a fold change $>1.5$ and a false discovery rate of less than $5 \%$ were detected in patients versus controls. Upregulated coding genes clustered in immunological, cell adhesion and keratin-related processes as previously found by microarray studies. Interestingly, 676 deregulated noncoding genes were detected, 257 of which were classified as antisense genes. $42 \%$ of these antisense genes had a concurrent deregulated sense gene.
The majority of the sense-antisense genes had a similar effect sizes in an independent North American dataset with three genes (OTUD6B-AS1, CTBP1AS2 and HMGN3-AS1) exceeding the study-wide Bonferroni-corrected $\rho$-value $\left(\mathrm{P}_{\mathrm{Bonf}}<0.0024, \mathrm{P}_{\text {combined }}=1.6 \times 10^{-9}, 1.7 \times 10^{-6}, 2.6 \times \times 10^{-6}\right.$, respectively). Intriguingly, the correlation of sense-antisense gene pairs deregulated in SSc is stronger than sense-antisense gene pairs not deregulated in SSc $(p<0.001)$.

Conclusions: For the first time we highlight that together with coding genes, (antisense) long noncoding RNAs are deregulated in skin tissue of SSc patients suggesting a novel class of genes involved in pathogenesis of SSc.

Disclosure of Interest: None declared

DOI: 10.1136/annrheumdis-2017-eular.6165

\section{FRI0357 MICROPARTICLES AS A BIOMARKER AND A REDOX-DEPENDENT REGULATOR OF NEUTROPHIL ACTIVATION AND PROTEOLYTIC ACTIVITY IN PATIENTS WITH SYSTEMIC SCLEROSIS}

N. Maugeri, P. Rovere Querini, S. Franchini, E. Tombetti, G. Ramirez, V. D’Alberti, M.G. Sabbadini, M. Bianchi, C. Lunardi, A. Gabrielli, A. Manfredi. AILS Patient Association on Scleroderma, Milan, Italy

Background: Persistent oxidative stress and unrelenting vascular inflammation are hallmarks of Systemic Sclerosis (SSc). Platelet-derived microparticles (PDUP) that express a bioactive redox-dependent moiety, HMGB1 accumulate in the peripheral blood of patients with SSc.

Objectives: To verify whether PD-uP might represent a biomarker of SSc clinical involvement and whether their biological actions is regulated by environmental redox.

Methods: Fifty-four patients with SSc were enrolled so far. Twenty healthy controls $(\mathrm{HC})$ matched for sex and age were studied in parallel. PD-uP were characterized and quantified by flow cytometry. Leukocyte features, including expression and distribution of myeloperoxidase (MPO), were assessed by flow cytometry and confocal microscopy. PD-uP ability to regulate neutrophil activation and proteolytic action was assessed in vitro in defined redox conditions and in vivo upon intravenous (i.v.) injection in immunocompromised NSG mice, and traced at various times based on recognition of the human platelet antigen CD61. Action on fibroblasts derived from SSc patients and $\mathrm{HC}$ are being assessed using biochemical and functional assays.

Results: PD-uP are present in the blood of SSc patients. Their concentration is significantly higher than in the blood of $\mathrm{HC}(p<0.0001)$. A substantially higher fraction of SSc PD-uP express the prototypic DAMP, HMGB1 $(70 \%$ SSc vs $5 \% \mathrm{HC}$ ). Among SSc patients, those with pulmonary hypertension had a significantly higher concentration of HMGB1+ PDuP $(p=0.002)$. In contrast other disease-associated variables, including the extent of fibrosis and the presence of active SSc pattern at the NVC, were not apparently influent. Neutrophils of SSc patients were activated, as demonstrated by the MPO redistribution from the primary granules to the plasma membrane. Moreover, they had a substantially higher ability to degrade fibrin in vitro, suggesting that enzymes at the plasma membrane are bioactive. Circulating neutrophils appeared to be viable and the fraction of cells undergoing apoptosis was similar in SSc patients and HC. The extent of neutrophil activation was associated with the concentration of HMGB1+ PDuP $(p<0.001)$. SSc PDuP but not HC PDuP induced MPO redistribution in vitro. The effect was dependent on HMGB1 and increased by oxidizing moieties. Injection in immunocompromised mice resulted in time-dependent association of SSc PDuP to mouse neutrophils, which contextually redistributed MPO at the plasma membrane.

Conclusions: HMGB1 expression on PD-up of SSc patients could help identify functionally relevant population of microparticles, involved in neutrophil activation/function and possibly valuable as a novel biomarker of vascular remodeling. References:

[1] Sabbadini MG et al, Lupus, 1999.

[2] Filippi et al., Lancet Neurol 2013.

[3] Rocca MA et al., Brain Struct Funct, 2016.

Disclosure of Interest: None declared

DOI: 10.1136/annrheumdis-2017-eular.3980

\section{FRI0358 99MTC-RHANNEXIN V-128 AS A NOVEL EARLY DIAGNOSTIC MARKER FOR INTERSTITIAL LUNG DISEASE ASSOCIATED WITH SYSTEMIC SCLEROSIS}

L. Guo ${ }^{1,2}$, J. Schniering ${ }^{1}$, R. Schibli ${ }^{3}$, A. Blanc $^{3}$, D. Chicco ${ }^{4}, \mathrm{~S} \mathrm{Ye}^{2}, \mathrm{O}$. Distler ${ }^{1}$, M. Béhé ${ }^{3}$, B. Maurer ${ }^{1}$. 'Department of Rheumatology, University Hospital Zurich, Zurich, Switzerland; ' 2 Department of Rheumatology, Renji Hospital, Shanghai Jiao Tong University, Shanghai, China: ${ }^{3}$ Center for Radiopharmaceutical Sciences, Paul Scherrer Institute, Villigen-PSI, Switzerland; ${ }^{4}$ Advanced Accelerator Applications, Colleretto Giacosa, Italy

Background: Interstitial lung disease (ILD), the primary cause of death in systemic sclerosis (SSc), often occurs early in the disease course, yet only becomes symptomatic when there is already substantial functional impairment and morphologic changes. Thus, there is an unmet need for early diagnosis. Apoptosis is considered the first pathophysiologic event in SSc-ILD. Monitoring of apoptotic processes with nuclear imaging, a sensitive, specific and noninvasive 
methodology, might be a promising new approach for the diagnosis of early SSC-ILD.

Objectives: To evaluate the radiotracer ${ }^{99 m}$ Tc-rhAnnexin V-128, which specifically targets a pathophysiologic key molecule of early apoptosis, for the detection of earliest stages of lung involvement in animal models of SSc-ILD with single photon emission computed tomography (SPECT/CT).

Methods: C57BL/6J mice were treated with a single intratracheal injection of bleomycin or saline. Animals were euthanized at days 3, 7, 14 and 21 postinjection $(n=6)$. Lung injury was evaluated by analysis of HE and Sirius red staining. The Ashcroft score was applied for the semi-quantitative evaluation of fibrotic changes. Immunofluorescence using the TUNEL assay and double staining with specific cell markers were performed to determine apoptotic cells. Positive nuclei were quantified by manual and automatic counting with Image $\mathrm{J}$ analysis software. Three days after injection with bleomycin or saline, mice were injected with 99m Tc-rhAnnexin V-128 (Advanced Accelerator Applications, Italy). After $1 \mathrm{~h}$, images were acquired using small animal SPECT/CT, followed by ex vivo autoradiography.

Results: In the model of bleomycin-induced lung fibrosis, inflammatory infiltrates (CD45+) occurred as early as day 3 with peak at day 7 , whereas pulmonary fibrosis developed from day 7 as assessed by Sirius red staining and was most pronounced at day 21 (mean Ashcroft score=4.6, $p=0.0286$ ). Notably, the number of apoptotic cells evaluated by TUNEL staining, was highest at day 3 (mean $\pm \mathrm{SE}=6.5 \pm 1.5$ positive cells/HPF, $p=0.0436$ ) compared with saline controls (mean $\pm \mathrm{SE}=0.7 \pm 0.1, p=0.0095$ ) and then decreased over time. To determine the type of apoptotic cells, we performed immunofluorescent co-stainings with different cell markers. Data displayed that endothelial cells ( $V W F+)$ and epithelial cells (cytokeratin+), but not inflammatory cells (CD45+) were the primary cells undergoing apoptosis in earliest inflammatory stages of ILD.

In accordance with the findings on tissue level, at day 3 post-injection, we detected ex vivo with autoradiography, yet not with in vivo SPECT/CT, an increased pulmonary uptake of ${ }^{99 \mathrm{~m} T c-r h A n n e x i n ~ V-128}$ in the lungs of bleomycininduced mice compared with saline treated controls.

Conclusions: Apoptosis of epithelial and endothelial cells preceded the development of pulmonary inflammation and fibrosis in the model of bleomycin-induced lung fibrosis. Thus, the use of ${ }^{99 m}$ Tc-rhAnnexin V-128 might be a promising approach for the diagnosis of earliest stages of ILD. However, sensitivity of in vivo imaging has to be further improved.

Disclosure of Interest: L. Guo: None declared, J. Schniering Grant/research support from: Swiss National Science Foundation (S-85605-02-01), R. Schibli: None declared, A. Blanc: None declared, D. Chicco Employee of: Advanced Accelarator Applications, S. Ye: None declared, O. Distler Grant/research support from: Actelion, Bayer, Boehringer Ingelheim, Pfizer, Sanofi;patent licensed mir-29 for the treatment of systemic sclerosis, Consultant for: 4 D Science, Actelion, Active Biotec, Bayer, Biogenldec, BMS, Boehringer Ingelheim, ChemomAb, EpiPharm, espeRare foundation, Genentech/Roche, GSK, Inventiva, Lilly, medac, Mepha, Medlmmune, Mitsubishi Tanabe Pharma, Pharmacyclics, Pfizer, Sanofi, Serodapharm, Sinoxa, Speakers bureau: AbbVie, iQone Healthcare, Mepha, M. Béhé: None declared, B. Maurer Grant/research support from: AbbVie, Protagen, EMDO, Novartis, Pfizer, Roche, Actelion. Patent licensed: mir-29 for the treatment of systemic sclerosis

DOI: 10.1136/annrheumdis-2017-eular.4945

\section{FRI0359 THE IMPACT OF NARROWBAND ULTRAVIOLET A1 ON PROLIFERATION AND APOPTOSIS MARKERS IN ANIMAL MODEL OF SCLERODERMA}

D. Karpec ${ }^{1,2}$, R. Rudys ${ }^{2}$, L. Leonaviciene ${ }^{2}$, Z. Mackiewicz ${ }^{2}$, R. Bradunaite ${ }^{2}$, G. Kirdaite ${ }^{2}$, R. Rugiene ${ }^{1,2}$, A. Venalis ${ }^{1,2}$. ${ }^{1}$ Center of Rheumatology, Vilnius University; ${ }^{2}$ State Research Institute Centre for Innovative Medicine, Vilnius, Lithuania

Background: Narrowband ultraviolet A1 (UVA1) phototherapy implications for systemic sclerosis still remain the area of research.

Objectives: To define the efficacy and safety of $365 \pm 5 \mathrm{~nm}$ UVA1 for the dermal fibrosis treatment in bleomycin-induced mouse model of scleroderma.

Methods: DBA/2 strain mice were randomly divided to 6 groups: I - healthy animals; II - control group with bleomycin induced scleroderma, III and IV - mice with established scleroderma, treated with high and medium dose of UVA1, V and VI - healthy mice, treated with high and medium dose of UVA1. Scleroderma model was induced according to the protocol [1]. Light source emitting a narrowband UVA1 of $365 \pm 5 \mathrm{~nm}$ and of $21 \mathrm{~mW} / \mathrm{cm}^{2}$ power density was used in the study. Phototherapy was performed 3 times weekly for 5 weeks. The average cumulative doses were $1200 \mathrm{~J} / \mathrm{cm}^{2}$ for high and $600 \mathrm{~J} / \mathrm{cm}^{2}$ for medium dose treatments. Histological analysis with hematoxylin-eosin staining for dermal thickness measurement was performed. The immunohistochemical staining for p53, Ki-67 and active caspase-3 proteins was performed using specific antibodies. Statistical significance was expressed by a $P$ value $<0.05$.

Results: The dermal thickness of mice treated with high and medium dose of
UVA1 was significantly reduced to $272.9 \pm 113.2$ and $394.0 \pm 125.9 \mu \mathrm{m}$, respectively, in comparison to the control group II $(599.0 \pm 55.7 \mu \mathrm{m})$. The percentage of Ki-67 positive cells in mice with scleroderma after high- and medium-dose of UVA1 did not differ from the control group (II). The expression of p53 was significantly higher in the skin of the control group (II) compared to that of healthy mice skin (group I). After treatment of mice with scleroderma with high- and medium-dose of UVA1, the expression of p53 in the dermal layers did not differ from the control group (II) of non-treated mice. There was no change of p53 nor Ki-67 expressions between healthy (group I) and UVA1-treated healthy mice skin (groups V and VI). The statistically significant increase of active caspase-3 expression in the skin of mice with scleroderma was present after high- and medium-dose of UVA1 (groups III and IV) as compared to that of non-treated mice group (II). The expression profile of active caspase-3 did not differ between healthy (group I) and UVA1-treated healthy mice skin (groups $\mathrm{V}$ and $\mathrm{VI}$ ). Results are summarized in Table 1 (Ki-67) and Figure 1 (A - active caspase-3; B - p53 immunohistochemical analysis).

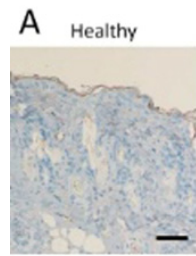

Bleo

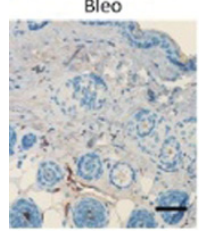

B Healthy

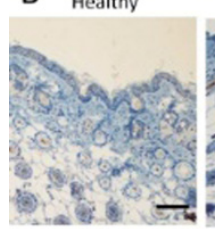

Bleo

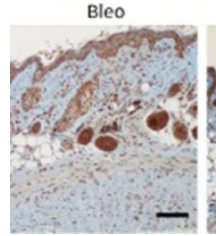

HealthyHighPT

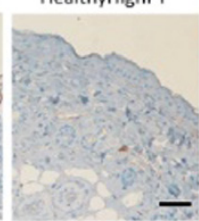

BleoHighPT

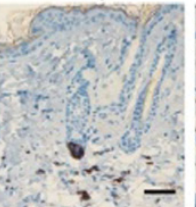

HealthyHighPT

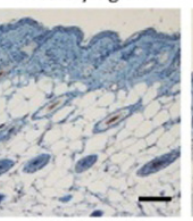

BleoHighPT

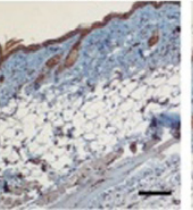

HealthyMediumPT

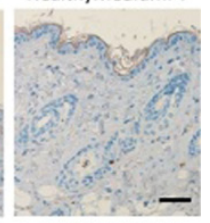

BleoMediumPT

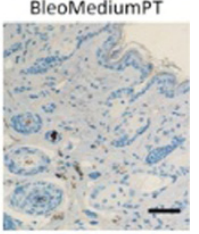

Conclusions: The cumulative doses of $1200 \mathrm{~J} / \mathrm{cm}^{2}$ and $600 \mathrm{~J} / \mathrm{cm}^{2}$ of narrowband UVA1 effectively reduced the dermal thickness, and the impact was dosedependent. Phototherapy course did not up-regulate p53 nor Ki-67 proteins in the healthy mice and mice with scleroderma skin. UVA1 radiation caused the increase of the active caspase- 3 expression in the skin of mice with scleroderma reflecting the apoptotic feature of narrowband UVA1. The results of this study indicate that $365 \pm 5 \mathrm{~nm}$ UVA1 phototherapy is safe and effective for the treatment of dermal fibrosis.

References:

[1] Avouac J. Mouse model of experimental dermal fibrosis: the bleomycin-induced dermal fibrosis. Methods Mol Biol 2014; 1142: 91-8.

Disclosure of Interest: None declared

DOI: 10.1136/annrheumdis-2017-eular.2869

\section{FRI0360 ANALYSIS OF ENDOCANNABINOID SYSTEM ELEMENTS AND RELATED INFLAMMATORY MOLECULES IN PERIPHERAL BLOOD LEUKOCYTES OF PATIENTS WITH SYSTEMIC SCLEROSIS}

C. Perez-Sanchez, R. Ortega-Castro, C. del Río, M.A. Aguirre, P. Ruiz-Limon, N. Barbarroja, Y. Jimenez-Gomez, I. Arias-de la Rosa, M.C. Abalos-Aguilera, E. Collantes-Estevez, E. Muñoz, C. Lopez-Pedrera. IMIBIC/Reina Sofia University Hospital/University of Cordoba, Cordoba, Spain

Background: The Endocannabinoid system (ECS) is a potential target for treatment of systemic sclerosis (SSc). Several cytokines/chemokines have been implicated in the induction of fibrosis in SSc, but their profile in peripheral blood

Abstract FRI0359 - Table 1

\begin{tabular}{|c|c|c|c|c|c|c|}
\hline Percentage of Ki-67 positive cells & Healthy (I) & Bleo (II) & BleoHighPT (III) & BleoMediumPT (IV) & HealthyHighPT (V) & HealthyMediumPT (VI) \\
\hline Average (\%) & 50.4 & 36.1 & 35.4 & 37.2 & 50.1 & 49.4 \\
\hline SD & 2.6 & 3.0 & 3.2 & 2.8 & 2.0 & 1.4 \\
\hline
\end{tabular}

